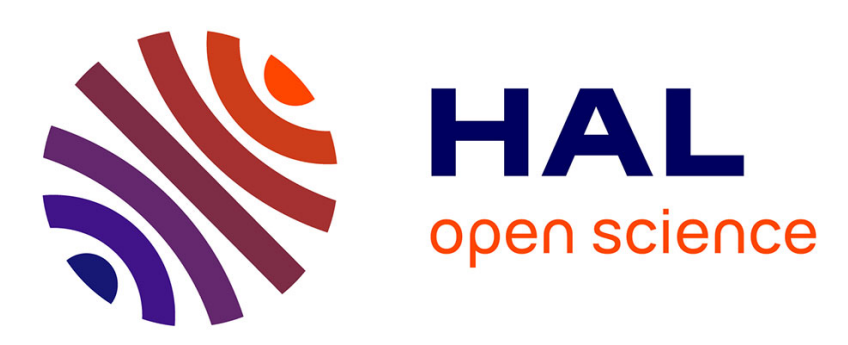

\title{
High-precision regulation of a pressure controlled artificial mouth: the case of recorder-like musical instruments
}

Didier Ferrand, Christophe Vergez, Benoît Fabre, François Blanc

\section{- To cite this version:}

Didier Ferrand, Christophe Vergez, Benoît Fabre, François Blanc. High-precision regulation of a pressure controlled artificial mouth: the case of recorder-like musical instruments. Acta Acustica united with Acustica, 2010, 96 (4), pp.701-712. 10.3813/AAA.918324 . hal-00445632

\section{HAL Id: hal-00445632 \\ https://hal.science/hal-00445632}

Submitted on 10 Jan 2010

HAL is a multi-disciplinary open access archive for the deposit and dissemination of scientific research documents, whether they are published or not. The documents may come from teaching and research institutions in France or abroad, or from public or private research centers.
L'archive ouverte pluridisciplinaire HAL, est destinée au dépôt et à la diffusion de documents scientifiques de niveau recherche, publiés ou non, émanant des établissements d'enseignement et de recherche français ou étrangers, des laboratoires publics ou privés. 


\title{
High-precision regulation of a pressure controlled artificial mouth : the case of recorder-like musical instruments
}

\author{
D. Ferrand ${ }^{\mathrm{a})}$ and Ch. Vergez ${ }^{\mathrm{b}}$ ) \\ Laboratoire de Mécanique et d'Acoustique, CNRS UPR 7051 \\ 31 Chemin Joseph Aiguier, 13402 Marseille Cedex 20, France \\ B. Fabre ${ }^{\mathrm{c})}$ and F. Blanc ${ }^{\mathrm{d})}$ \\ IJLRD, UMR CNRS 7190, UPMC, \\ 4 Place Jussieu, 75252 Paris Cedex 05, France
}

(Dated: January 10, 2010)

\begin{abstract}
Real-time regulation of air pressure in an artificial mouth is studied. A closed-loop strategy is developped, including a controller algorithm which updates (according to the difference between the target and the measured blowing pressure) the electrical command sent to the sero-valve providing air flow. In this paper the targets considered may be steady states or slowly varying trajectories. Since both the musical instrument and the servo-valve behave nonlinearly, the design of the controller algorithm requires preliminary modelling and identification steps in order to optimize the compromise performances/robustness. The controller finally adopted for the experiments is a PI (Proportional Integral) whose integral gain is adapted according to an online estimator. Moreover, a carefully tuned dither signal is added on the electric command in order to limit consequences of friction inside the servo-valve. The high-precision regulation is used to investigate the functionning of the recorder. Firstly, it allows to highlight the existence of aeolian sounds which had never been observed on a recorder. Moreover, the influence of some small "details" of the chamfers geometry on the global behavior of the instrument is investigated. Hence the influence of the maker's work can be evaluated, as well as the important consequences on the possibilities offered to the player: with the optimized chamfers, the instrument can sound louder and the transition between the two regimes is safer (larger hysteresis width).
\end{abstract}

PACS numbers: 43.75.Ef, 43.75.Qr, 43.75.Yy

\section{INTRODUCTION}

Blowing machines (or artificial mouths), were developed since the early stage of research in musical acoustics (at least since 65 years ${ }^{1}$ ) because they are necessary for the study of musical instruments. When replacing a musician by an artificial mouth, what is seeked is obviously more controlled measurements, since the different parameters may be tuned independently by the experimeter. The blowing pressure is usually tuned by hand through a pressure reducer, which may become a very tricky task when a precise value of the blowing pressure is wanted, or an even impossible challenge when regular ramps are required to measure very precisely bifurcations. Without speaking about the inevitable perturbations linked for example to fluctuations of the pressure generator, or of the pressure reducer.

Therefore, we focus in this paper on the high-precision

a) Electronic address: ferrand@lma.cnrs-mrs.fr

b) Electronic address: vergez@lma.cnrs-mrs.fr

c) Electronic address: fabreb@ccr.jussieu.fr

d) Electronic address: blanc@lam.jussieu.fr regulation of the air pressure in an artificial mouth coupled to a recorder. What is meant by regulation is the control of the blowing pressure around a fixed value or around a value whose evolution in time is quasi-static (like slowly varying ramps). It is therefore a different problem from the reproduction of patterns recorded in the mouth of actual musicians. This would require a pressure control with transients as fast as a few milliseconds, which is out of the scope of this paper. Our goal is not here to mimic an instrumentalist playing an instrument but to provide a better control in experiments with artificial mouths.

The specifications for the control will be detailed in section II, as well as the motivations to developp a closedloop strategy. This approach requires the modelling and the identification of the experimental setup (section III) in order to design an adapted control architecture (section IV). Then, we demonstrate some examples of experiences with a recorder where the contribution of the high-precision regulation is decisive (section $\mathrm{V}$ ). 


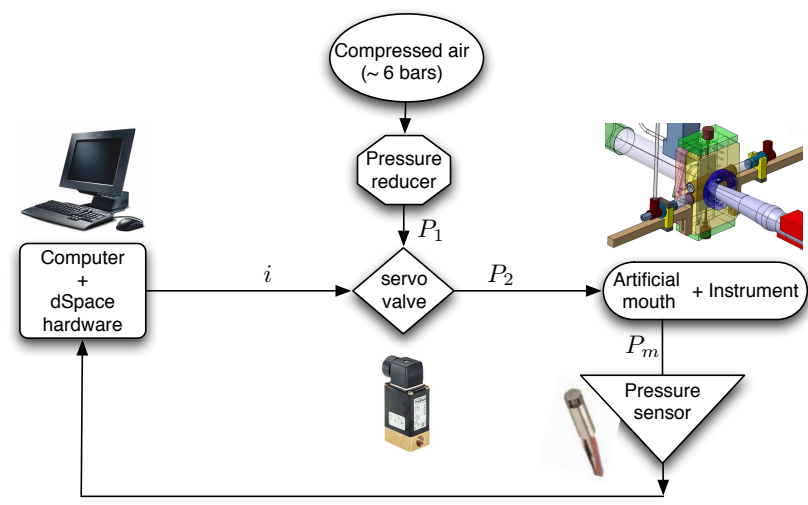

Figure 1. Principle of the pressure controlled artificial mouth. Through a control algorithm implemented on a DSP card, the volume flow through the servo-valve is modified every $40 \mu \mathrm{s}$ in order to minimize the difference between the measured and the target mouth pressure.

\section{PROBLEM STATEMENT}

\section{A. Experimental setup}

The experimental setup considered in this paper is presented in figure 1. A servo-valve is connected to compressed air through a pressure reducer. The maximum pressure available is around 6bars, and the pressure reducer is used to adjust the pressure $P_{1}$ upstream the servo-valve. The servo-valve is connected at the entrance of the artificial mouth itself (whose $30 \mathrm{~cm}^{3}$ internal volume is the place where the air pressure $P_{m}$ must be controlled). The artificial mouth blows into the recorder.

The air flow through the servo-valve depends on the position of an internal moving armature. This position is controlled by the electrical current $i$ sent to the servovalve through a linear power amplifier (the original integrated PWM electronics of the servo-valve has not been used since it was too noisy for acoustic measurements). The choice of a proportional servo-valve has been done on the basis of existing industrial air regulators designed around a servo-valve electronically controlled according to a pressure sensor at the oulet. Indeed, since the pressure to control is inside the artificial mouth cavity, separate servo-valve and pressure sensor are required and have been associated within a closed loop control scheme, as suggested by figure 1 (details on the motivation for a closed-loop control are given in section II C).

The commands are numerically coded on a dSpace fast prototyping development system: $40 \mu \mathrm{s} / 16$ bits ADC (Analog to Digital Converter) for pressure acquisitions, 16 bits $/ \pm 10 \mathrm{~V}$ DAC (Digital to Analog Converter) for command of the servo-valve through a linear power amplifier (providing a regulated current from 0 to $400 \mathrm{~mA}$ from a +/- 10V DAC command).

\section{B. Specifications for the control}

The pressure $P_{m}$ must be controlled within the cavity. Compared to the classical use of an artificial mouth where the blowing pressure is tuned by hand through the pressure reducer, the aim is to increase both precision, stability and rapidity with respect to various kinds of targets : constant or slowly increasing/decreasing (ramps). Fast time-varying targets are a specific problem and are not considered in this paper.

The aim is to be able to control both slight variations of the blowing pressure as well as to sweep a large range. In the musical acoustics context, this correspond to a zoom on the instrument behavior around a given blowing pressure (for example to detect a bifurcation), or on the contrary to the study of its global behavior when the blowing pressure is swept over the whole playing range.

When considering constant target pressures, the asymptotic error should not go beyond a few Pascals. This objective is required since we want to measure thresholds of auto-oscillation, or bifurcations between registers very precisely. The specification on the control performances (time response, bandwidth) shall mainly depend on disturbances identification (external, such as air pressure supply fluctuations, or internal, such as friction inside the servo-valve) and trajectory requested performances (static errors, time response to reach the trajectory).

Depending on the instrument and on the playing nuance considered, blowing pressure varies for many orders of magnitudes. Regarding the recorder, typical blowing pressures vary from tens of $\mathrm{Pa}$ to at most a few $\mathrm{kPa}$ for upper registers.

\section{Why close the loop ?}

The open-loop approach would consist in sending a voltage reference to the servo-valve electronics without any feedback of the result. This would require exact knowledge of the process to hit the desired result, which is not reachable.

"Closing the loop" means that a system with transfer function $H(s)$ is replaced by a structure described in figure 2. In our case, $H(s)$ represents the servo-valve, the artificial mouth and the recorder. Closing the loop allows to control the process only on the basis of a measurement, without the need of the knowledge of the whole process.

More precisely, the closed loop tends to transform a process $H(s)$ with input $x(s)$ and output $y(s)$ into a new system (input $X(s)$, output $Y(s)$ ) with same input and output physical unit (here expressed in $\mathrm{kPa}$ ) and a new transfer function (the so-called closed-loop transfer function):

$$
G(s) \triangleq \frac{Y(s)}{X(s)}=\frac{C(s) H(s)}{1+C(s) H(s)},
$$

where $C(s)$ is the transfer function of the digital con- 


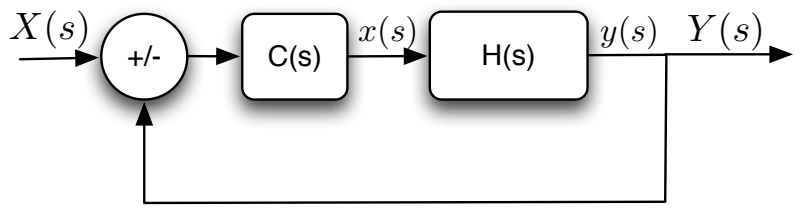

Figure 2. Prototype of a closed loop : $H(s)$ and $C(s)$ are the transfer functions of the process and of the controller respectively. The control hopefully converges through an interative procedure with update based on the measurement of the error.

troller which converts the error into a new command sent to the system.

The basic idea of control is that if the gain $|C(s) H(s)|$ is far bigger than unity on the whole frequency range, then $|G(s)|$ tends toward unity, which is the goal to achieve: to control a measured output according to an action on the input. The gain $|C(s) H(s)|$ is important in terms of performance of the control and that's the role of the controler to provide practically additional gain (at least before a cutoff frequency). On the other hand the possible existence of poles, i.e. roots of

$$
1+C(s) H(s)=0,
$$

shows that this system may become unstable, if for some $s, C(s) H(s)$ yields some gain larger than 1 with $-180^{\circ}$ phase shift. That is why tuning the controller is a matter of compromise between performances of the control and stability. The open-loop study (i.e. frequency responses of $C(s) H(s)$ ) shall indicate these performance and stability characteristics (see section IV A 2 and IV A 3).

Since the closed-loop approach only appears as a compromise, it is worth insisting why its alternative, the open-loop approach, has not been retained. Indeed, its two main advantages are: no stability issue and no influence of the downstream load on the control. However since the process and the actuator are non linear (e.g. friction within the servo-valve), it is very difficult to "guess" which current should be sent to the servo-valve

- In the case of static targets, important static errors are likely to occur. This can be adjusted by hand obviously, but this way of doing would not present any advantage compared with the traditional use of an artificial mouth (blowing pressure adjusted by hand through a pressure reducer). Moreover, external disturbances would result in fluctuations of the pressure around the desired target.

- When a linear ramp is wished, errors are not so crucial. More important is the regularity of the obtained increase in pressure. The major problem is that a linearly increasing electrical command will result in a blowing pressure increasing non regu-

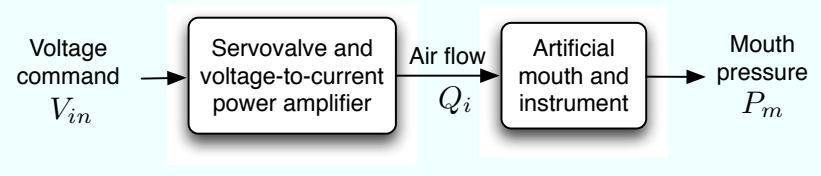

Figure 3. Synoptic of the actuator and process.

larly. This corresponds to the alternance of stick and slip phases inside the servo-valve.

To cope with that problem, some authors $\left({ }^{2}\right)$ propose to identify a friction model in order to precompensate for friction. However in this paper, a closed-loop approach has been prefered.

\section{MODELLING / IDENTIFICATION OF THE EXPERIMENTAL SETUP}

The aim of this stage (modelling and identification) is then to be able to study the control capabilities in terms of best control algorithm and related foreseen performances.

The first step toward the definition of the controller $C(s)$ is to model the actuator and the process to be controlled. In our case, the actuator is the servo-valve, and the process is the artificial mouth plus the recorder. This is done through simple assumptions on the physics of the process functionning (modelling step), to be further confirmed by actual measurements to identify the physical parameters of the model (identification step).

Since the control of the mouth pressure $P_{m}$ should be performed by a voltage reference $V_{\mathrm{DAC}}$ through a DAC, the process is modeled through two main cascaded subsystems (see figure 3):

- the servo-valve transfer function between the volume flow $Q_{i}$ provided by the servo-valve and the voltage command $V_{\mathrm{DAC}}$.

- the articial mouth and instrument transfer function between the mouth pressure $P_{m}$ and the volume entering the artificial mouth $Q_{i}$.

These models are detailed herafter.

\section{A. The actuator}

The chosen actuator is a proportional servo-valve (Bürkert 2832 with a $1.2 \mathrm{~mm}$ outlet diameter) which claims to provide a volume flow proportional to the inlet electrical current. The servo-valve is controlled by a voltage to current linear power amplifier (maximum ouput current of $400 \mathrm{~mA}$ for a $10 \mathrm{~V}$ input voltage).

Various analysis and experiments which have been carried out reveal that the principle of functionning of the servo-valve can be mainly reduced to a moving armature 
(closing more or less a leakage aperture) driven by several external forces: an electromagnetic force proportionnal to a current (the electric command), a spring force (with offset due to taring), a pressure force (due to air supply) and a friction force. Therefore, three different aspects of the servo-valve functionning are considered for the transfer function modelling:

- The fluid dynamics, i.e. the relation between the volume flow $Q_{i}$, the channel opening $x$ and the pressure drop across the servo-valve $P_{1}-P_{2}$ (see sections III A 1 and III A 2).

- The armature own dynamics, mainly in terms of resonance frequency and damping ratio (see section III A 3).

- The internal friction (see section III A 4).

\section{Fluid modelling}

According to the technical datasheet of the servo-valve, the volume flow $Q_{i}\left(\mathrm{~m}^{3} / \mathrm{h}\right)$ can be calculated according to the channel opening $x$ (corresponding to the armature position, $x=0$ (resp. $x=1$ ) corresponding to the closed (resp. fully opened) channel) and to the pressure drop across the servo-valve:

$$
\begin{array}{ll}
Q_{i}=514 k_{v} x \sqrt{\frac{P_{2}\left(P_{1}-P_{2}\right)}{T \rho}} & \text { if } P_{1} \leq 2 P_{2} \\
Q_{i}=\frac{257}{\sqrt{T \cdot \rho}} P_{1} k_{v} x & \text { if } P_{1}>2 P_{2}
\end{array}
$$

where $P_{1}$ and $P_{2}$ are the absolute pressures (given in $\mathrm{Pa}$ ) at the inlet and at the outlet of the servo-valve respectively, $\rho$ is the air density $\left(\mathrm{kg} / \mathrm{m}^{3}\right), T$ is the absolute temperature $(K)$ and $k_{v}\left(\mathrm{~m}^{3} / h\right)$ is a given parameter. Eq. (3) and (4) are referred as the subcritical and supercritical regimes respectively. In our experiments, care is taken to check that the servo-valve has a supercritical behavior, since in that case the volume flow depends linearly on $P_{1}$ and is independent of the outlet pressure $P_{2}$, which is remarkably simple. Therefore, only Eq. (4) should be considered in the following.

According to the datasheet, the volume flow actually yielded by the servo-valve should also vary linearly from 0 to $Q_{\max }$ when the driving current is varied from 250 $\mathrm{mA}$ to $400 \mathrm{~mA}$. This will be checked in sections III A 2 and III A 4.

\section{2. $k_{v}$ parameter identification}

In order to identify the actual $k_{v}$ coefficient, the volume flow $Q_{i}$ through the servo-valve has been measured with a flow meter for different $P_{1}$ at maximum opening. Results are displayed in Fig. 4. A $k_{v}$ of 0.052 has been found instead of the one given in data sheets $\left(k_{v}=0.04\right)$. Two main reasons explain this difference. First of all, mechanical tolerances for a specific valve yields $k_{v}$ disparity.

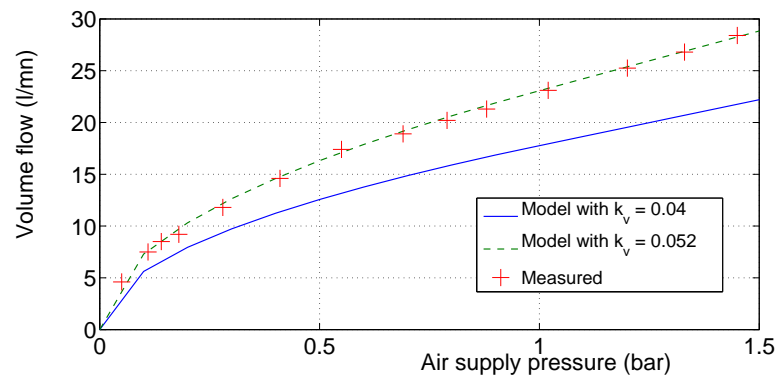

Figure 4. Volume flow $Q_{i}$ through the servo-valve at maximal opening when upstream relative pressure $\left(P_{1}-P_{a t m}\right)$ is varied. Measurements $(+)$, simulation with $k_{v}$ found on datasheets (dot-dashed) and $k_{v}$ identified on experimental data (dotted).

Secondly, the $k_{v}$ is given by the manufacturer for a usefull range of the valve (a fraction of the maximum possible opening) and not for a totally open operating point.

\section{Armature dynamics}

The moving armature itself is modelled as a mass/spring second-order system. The Laplace transfer function of the actuator is then:

$$
A(s)=\frac{\omega_{r}^{2}}{s^{2}+2 \xi \omega_{r} s+\omega_{r}^{2}}
$$

The resonance frequency is measured around $\omega_{r} / 2 \pi=$ $240 \mathrm{~Hz}$, the damping term is roughly estimated to $\xi=0.3$ according to various observations not detailed for sake of brevity.

\section{Inner friction and resulting hysteresis}

A friction force has also to be taken into account in the modelling. The precise modelling of the friction force is challenging and is out of the scope of this paper. For control analysis, friction is taken into account through a simplified phenomenological model consisting of an hysteresis (parameterized by the supply pressure $P_{1}$ ) applied on the electrical command. This model has been inspired by measurement of the volume flow $Q_{i}$ when the DAC command is varied slowly compared to the armature resonance frequency (see figure 5). The servo-valve starts to open for a value of the driving current which is all the smaller as $P_{1}$ is increased. This is due to the fact that a larger $P_{1}$ compensates a larger part of the taring force. The case $P_{1}-P_{a t m}=0.5$ bar shows a far less linear variation of $Q$ with respect to $P_{1}$ since the supercritical regime is not reached (Eq. (3) and (4)).

\section{Simplified complete model of servo-valve}

When gathering the information of sections III A 1 to III A 4, a servo-valve model can be proposed and will be used in section IV. 


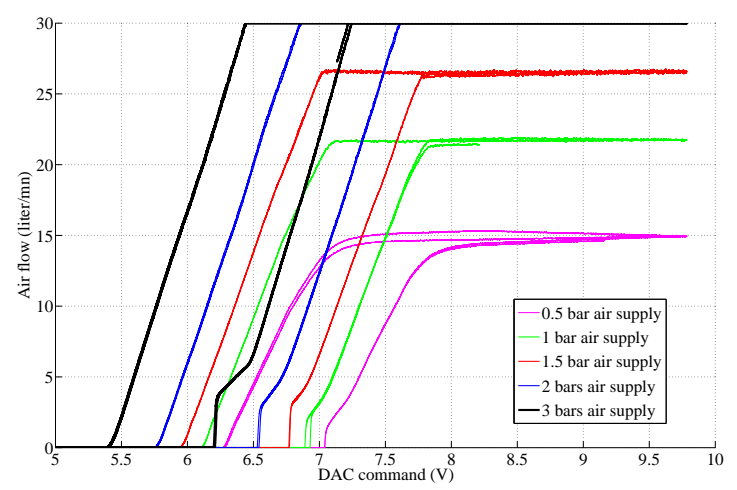

Figure 5. Volume flow $Q_{i}$ through the servo-valve measured for five upstream relative pressure values $P_{1}-P_{a t m}$ according to the driving current.

\section{B. The process: mouth volume and resistive effect of the instrument}

\section{Modelling : general case}

Let us recall that in this paper, the process is formed by the artificial mouth and the instrument. For sake of simplicity, a rather crude model is considered where the artificial mouth is reduced to its inner volume $V$ (artificial lips have not yet been taken into acount and are considered as a simple mechanical rigid mount), and the instrument by the pressure/volume flow quasistatic characteristics at the instrument inlet. In the general case, the volume flow $Q_{l}$ blown into the instrument depends non linearly on the mouth pressure $P_{m}$ :

$$
Q_{l}=f\left(P_{m}\right)
$$

The latter relation assumes that the static pressure inside the instrument is negligible compared to $P_{m}$, so that the pressure drop $P_{m}-P_{0} \simeq P_{m}$.

We are now interested in deriving a model of the (linearized) transfer function of the process, i.e. according to figure 3 an expression of the ratio between small fluctuations (around a static equilibrium) of the mouth pressure and of the volume flow entering the artificial mouth. Hence, $P_{m}, Q_{i}$ and $Q_{l}$ are written as (small) deviations around their steady values $P_{m_{0}}, Q_{i_{0}}$ and $Q_{l_{0}}$ :

$$
\begin{aligned}
P_{m} & =P_{m_{0}}+p_{m} \\
Q_{i} & =Q_{i_{0}}+q_{i} \\
Q_{l} & =Q_{l_{0}}+q_{l}
\end{aligned}
$$

Since according to Eq. (6) $Q_{l}$ is a function of $P_{m}, q_{l}$ can be written (at first-order in $p_{m}$ ):

$$
q_{l}=\left.\frac{d Q_{l}}{d P_{m}}\right|_{P_{m_{0}}} p_{m}
$$

Assuming the state equation for perfect gases:

$$
P_{m}=\frac{M \cdot R \cdot T}{V},
$$

where $R$ is the constant for a perfect gas, $M$ is the mass of air, $T$ is the temperature and $V$ is the volume of air considered. In this paper, $T$ and $V$ are assumed constants. The fact that the dynamics inside the mouth is considered isothermal (and not adiabatic) is motivated by the fact that we are interested in slow variations of pressure (linked with the goal of regulation around steady values).

At rest (i.e. when the system is at an equilibrium point), we have:

$$
Q_{i_{0}}=Q_{l_{0}}
$$

The (small) variation $\delta M$ of the mass of air inside the cavity, due to a (small) variation of incoming volume flow $q_{i}$ is:

$$
\delta M=\int \rho\left(q_{i}-q_{l}\right) d t
$$

where $\rho$ is the air density (which is considered constant since only small variations are considered).

\section{Modelling: recorder case}

In the case of a recorder instrument, and since the cross section $S$ of the channel at the tip of the instrument is constant, the relationship between volume flow and mouth pressure is approximated in the range of blowing used in traditionnal playing by a Bernoulli law (this is to be confirmed by measurements: see adaptive control section IV A 4). In this case:

$$
Q_{l}=\alpha \sqrt{P_{m}} \quad \text { with } \alpha=S \cdot \sqrt{2 / \rho} .
$$

For reed or brass instruments, the pressure applied on the reed/lips modifies the cross section through which air flows), hence $Q_{l}$ would have a more complicated expession.

Therefore, according to Eqs. (10) and (14):

$$
q_{l}=\frac{\alpha}{2 \sqrt{P_{m_{0}}}} p_{m}
$$

Therefore, according to Eq. (11) and Eq. (13) :

$$
\frac{d p_{m}}{d t}=\frac{1}{\nu}\left(q_{i}-\frac{\alpha}{2 \sqrt{P_{m_{0}}}} p_{m}\right),
$$

where $\nu=\frac{V}{\rho . R . T}$ is a constant. We now define $r_{i}$ as the differential air flow resistance:

$$
r_{i}=\frac{2 \sqrt{P_{m_{0}}}}{\alpha}
$$

Laplace transform of Eq. (16) leads to $\left(p_{m}(0)=0\right)$ :

$$
\frac{p_{m}(s)}{q_{i}(s)}=\frac{r_{i}}{1+r_{i} \nu s}
$$

This means that the process viewed from the entry of the mouth cavity behaves (under small perturbation) as 
a first order filter with gain $r_{i}$ and time constant $\tau=$ $\nu r_{i}$ depending on the operating point imposed by the instrument (through $P_{m_{0}}$ and $Q_{l_{0}}$ ). This highlights an inherent non linear behavior to cope with for the control scheme.

Attention of the reader is drawn on the fact that only the resistive quasi-static role of the instrument has been taken into account in this rough modelling (the acoustic response is not taken into account and considered as a second order parameter in the case of a recorder).

\section{Identification for small pressure fluctuations (regulation application)}

Measurements are carried out on a test case where the instrument is actually only represented by its resistive effect (an adjustable leak) and the artificial mouth is replaced by a rigid cavity. Two techniques may be used for parameter identification: step and frequency responses. Step responses have been preferred to frequency responses to avoid as much as possible the effect of friction on measurements, since the servo-valve is not part of the process to be identified.

In order to check the model (Eq. (18)) which foresees a variable flow resistance and time constant for a linearised model, step time responses have been measured for various operating points (pressure $P_{m_{0}}$ levels). To obtain this, unitary (electrical) steps are applied on the servovalve to produce constant variations of volume flow $q_{i}$ around different mean volume flow values $Q_{i_{0}}$ (see figure 6 ). Though it is already obvious that the amplitude of pressure steps increase according to the pressure level, a closer inspection reveals that the agreement with the model (Eq. (18)) is more than qualitative. Indeed in figure 7 , the amplitude of each step is plotted with respect to $P_{m_{0}}$, revealing the square-root dependance (accordingly to Eq. (17)). Moreover, in order to check wether the time constant is simply proportional to the step amplitude like in the model, the different steps of figure 6 are presented synchronized in time (figure 8, plain lines) superimposed with first-order filter responses with $r_{i}$ estimated from figure 7 and $\tau$ proportional to $r_{i}$. Agreement is probant, except for the first step for which the actual time constant is larger. Therefore, we conclude that Eq. (18) is a convincing model, which is used later for the synthesis of the controller.

\section{Complete transfer function}

The complete model (servo-valve plus artificial mouth coupled with the instrument) is represented by the following transfer function:

$$
H(s)=\frac{p_{m}(s)}{V_{\mathrm{DAC}}(s)}=\frac{r_{i}}{1+s \nu \cdot r_{i}} K_{s v} A(s)
$$

where $A(s)$ is the servo-valve transfer function given by Eq. (5), and $K_{s v}$ takes into account the slope of the

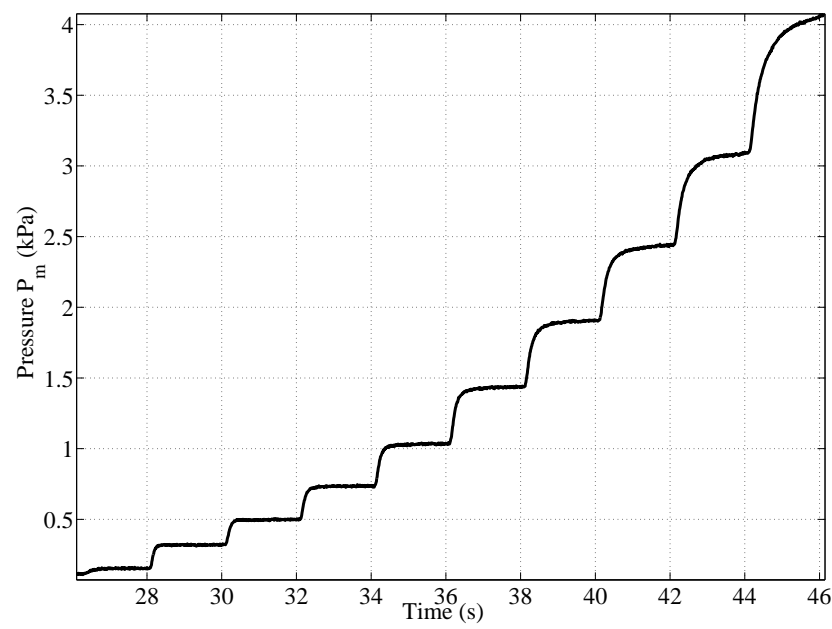

Figure 6. Pressure $P_{m}$ step responses at different pressure operating points for an unitary air flow step input (corresponding to the same increase in the voltage command)

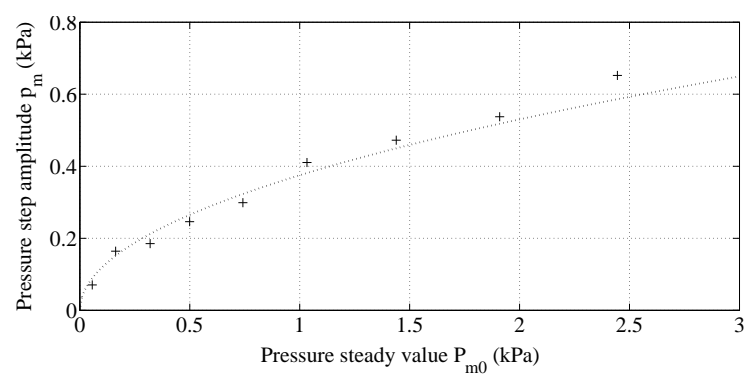

Figure 7. Same data as in figure 6 but the amplitude of each step is plotted with respect to $P_{m_{0}}(+)$ revealing a square-root dependance (dotted line) accordingly to Eq. (17).

servo-valve characteristics (figure 5) where the servovalve is assumed to operate in its linear range. This model should be considered as the linear part of the complete model. Due to friction indeed, some gain as well phase delay must be added to the model. These additional gain and phase may be estimated using first harmonic approach (as it has been done $\mathrm{in}^{8}$ ).

Figure 10 shows the frequency response of the model (Eq. (19)) at two different operating points $\left(P_{m}=10 \mathrm{~Pa}\right.$ and $P_{m}=3 \mathrm{kPa}$ ), showing different low frequency gains (25 dB of difference) as well as different cut off frequency of the system due to the pole (barely discernable on the gain but more obvious on the phase due to the $90^{\circ}$ phase delay). At $240 \mathrm{~Hz}$ we see the cut off frequency due to servo-valve dynamics with an additional phase lag of 180 ${ }^{\circ}$ and a resonance on the amplitude.

\section{HIGH-PRECISION REGULATION}

Let us recall that what is meant by regulation is the control of the blowing pressure around a fixed value or 


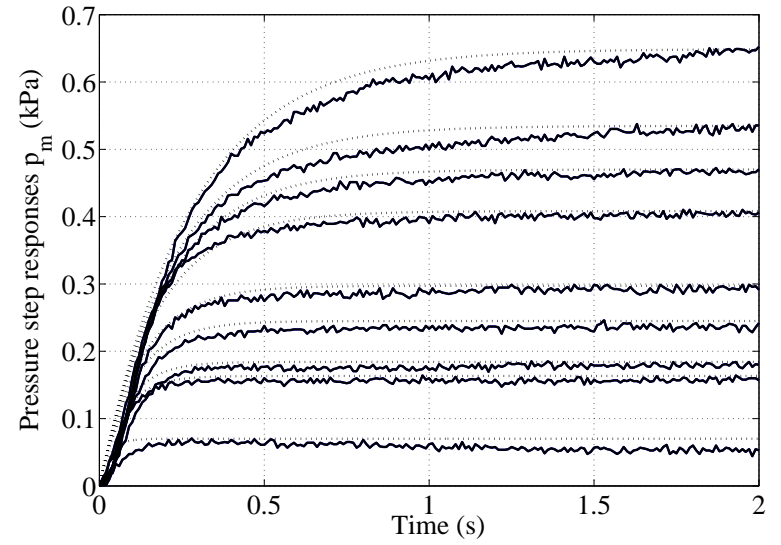

Figure 8. Same data as in figure 6 but time responses are presented synchronized (plain lines) with first-order filter responses (dotted lines) where the gain is eestimated from figure 7 and the time constant is chosen proportional to the gain.

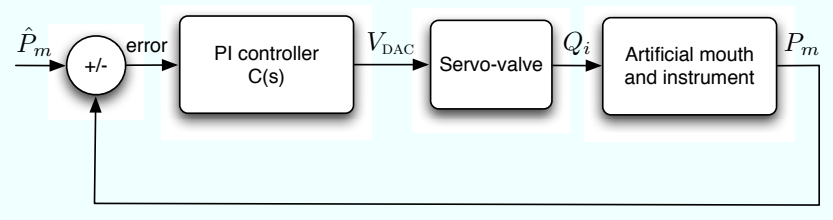

Figure 9. Principle of the pressure controlled artificial mouth. Through a control algorithm implemented on a DSP card, the volume flow through the servo-valve is modified every $40 \mu \mathrm{s}$ in order to minimize the difference between the measured and the target mouth pressure.

around a value whose evolution in time is quasi-static (slowly varying ramps).

\section{A. Closed-loop strategy}

\section{Principle}

In this paper, the closed-loop approach is adopted (see figure 9): the pressure $P_{m}$ measured in the artificial mouth is compared to the desired pressure (the target $\hat{P}_{m}$ ) and according to the error $e$ (the difference between the two values), the electric command (current $i$ ) sent to the servo-valve is altered. This is done each $40 \mu s$. The way of modifying the electric command $i$ according to the error $e$ depends on the choice of the controller, and is the main topic discussed throughout section IV A.

\section{Choice of the controller}

A Proportional Integral Derivative controller scheme (named PID hereafter) has been chosen as a first approach (and preferred to pole compensation, or other model-based optimal controllers) because:

- Both the actuator and the process have a nonlinear behavior, therefore a robust controller is necessary.

- The order of the process to control is rather low: typically, Eq. (19) shows a first-order system. Therefore, only a few degrees of freedom should be required for the controller (a PID controller has three degrees of freedom).

The electric command $i(t)$ sent to the servo-valve according to the error $e(t)$ is then:

$$
i(t)=k_{p} e(t)+k_{d} \dot{e}(t)+k_{i} \int e(t)
$$

where $k_{p}$ (resp. $k_{d}, k_{i}$ ) is the proportional (resp. derivative, integral) gain.

However, because of large gain fluctuations of the process according to the operating point (due to nonlinearity), refinements have been added to the controller. Nonlinear PID (which relies on non linear functions of $e(t),{ }^{?}$ ) has first been considered and evaluated. However, gain-scheduling techniques (pre-compensation of the PID gains with respect to a prediction according to the target) have been prefered $\left({ }^{?}\right)$ since it adresses the souce of the difficulty, i.e. the gain variation due to the nonlinearity. Finally, to overcome the limitations of pre-compensation techniques, an alternative approach has been retained (adaptive command) and is developped in section IVA 4. Moreover additional refinements are adeed to limit the drawbacks of the servo-valve inner friction (see section IV A 5).

\section{Tuning of the PID : compromise between performance and stability}

It has been shown in ${ }^{8}$ that after process and actuator modelling and parameter identification, the controller synthesis (PID tuning) can be performed using a first harmonic analysis in the Nichols plane. This analysis gives the stability conditions required to achieve the best compromise on the closed loop performance, between rapidity and stability. It appears that a major contribution on the instability is the effect of the hysteresis yielded by the actuator as well as the resonance of the servo-valve.

Indeed, it arises that $k_{p}$ cannot be increased at will in order to preserve the stability. Therefore, the resulting asymptotic static error (due to a relatively low $k_{p}$ ) is compensated by the integral term $k_{i}$. But again, since the integral term adds phase lag, the value of $k_{i}$ cannot be modified at will without reaching the instability region of the closed-loop.

In our application, we didn't use the derivative term. Indeed, it appears that since the error is very noisy (because $e(t)$ is formed according to a measured pressure with a significant amount of turbulent noise) the contribution $k_{d} e(t)$ to the electric command is always very noisy and finally, the choice $k_{d}=0$ has been done. Another drawback of the derivative term is its reinforcement of the mechanical mode of the servo-valve. The controller 


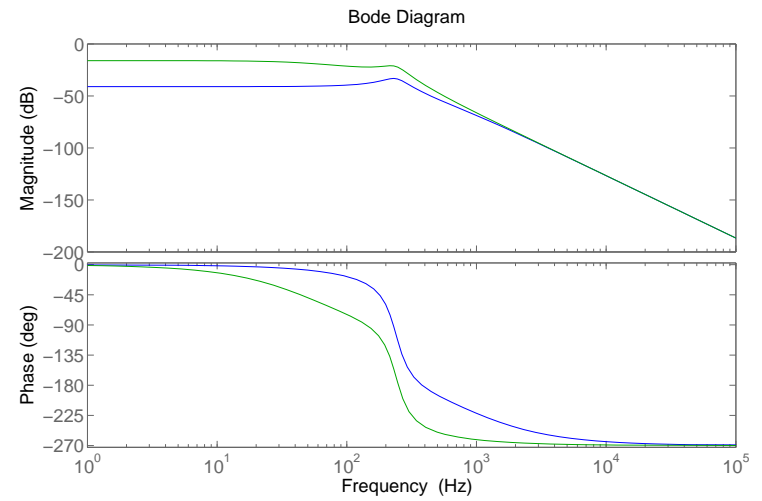

Figure 10. Process and servo-valve linearized transfer function at two different operating pressure point: $10 \mathrm{~Pa}$ (blue line) and $3 \mathrm{kPa}$ (green line).

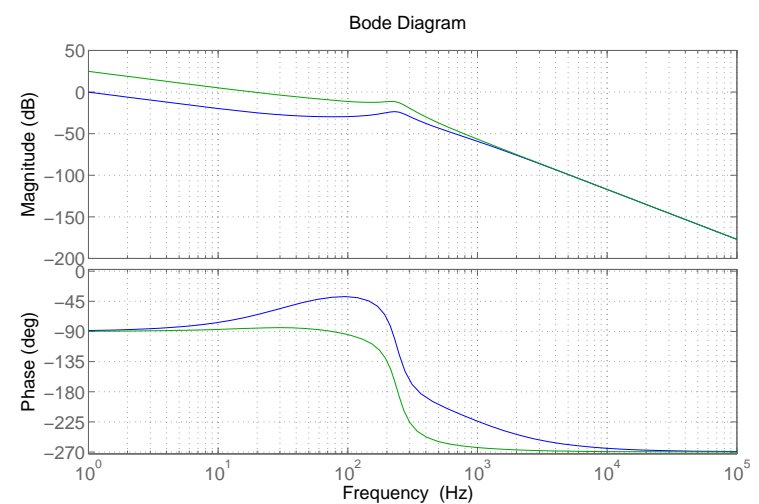

Figure 11. Frequency response (modulus and phase) of Eq. (22) (with $k_{p}=3, k_{i}=700$ ) for two operating points of the recorder: $10 \mathrm{~Pa}$ (blue line) and $3 \mathrm{kPa}$ (green line) .

which has been used was thus a simple PI controller with a transfer function:

$$
C(s)=\frac{k_{i}}{s}+k_{p}
$$

Consequently, the complete open-loop tranfer function including the PI controller and the process can be modelled as $T_{f}(s)=C(s) H(s)$ :

$$
T_{f}(s)=r_{i} \frac{k_{i}}{s} \frac{\left(1+\frac{k_{p}}{k_{i}} s\right)}{1+\nu r_{i} s} K_{s v} A(s)
$$

As seen on figure 11, the integral effect brings additional gain ar low frequencies ( compared to figure 10). The variable pole and gain yielded by the process shall modify the dynamical behavior as seen on the frequency responses for two different operating points $(10 \mathrm{~Pa}$ and $3 \mathrm{kPa})$.

a. Closed loop simulations : We now simulate the closed-loop response, that is, as seen in section II C, we consider the transfer function $\mathrm{G}(\mathrm{s})$ given by:

$$
G(s)=\frac{T_{f}(s)}{1+T_{f}(s)}
$$

Due to the different gains of the process at the two operating points associated with a single PI tuning resulting from a compromise, the closed loop time response shall be quite different for these two extreme cases (see figure 12).

\section{Adaptive command}

Previous paragraph showed that the control bandwidth, so the performance to follow precisely a pressure target, depends on the operating point due to the variable behaviour of the instrument. Indeed, if the controller is tuned correctly for low blowing pressures, the control loop may become unstable for high pressures. Conversely, if the controller is tuned to avoid instability at high pressures, the target following at low pressure is poor due to lack of gain in the system to operate an efficient control (simulated in figure 12, but similar experimental results have been observed).

In order to homogeneise the control performances, this section details the automatic tuning of the controller according to the operating point. Two questions have to be answered:

- How to determine the operating point in "real time" ?

- How should the parameters $k_{p}$ and $k_{i}$ be modified according to the operating point ?

a. Online estimator Ideally, the quantity to estimate should be the differential air flow resistance (Eq. (17)). But, since this estimation has to be done in real-time during a measurement, we cannot perturb the system at will (like in section III B 3). Therefore we assume that (like in the model) $r_{i}$ is proportional to $P_{m_{0}} / Q_{l_{0}}$. Hence the update of the controller coefficients will be based on the estimate of the total gain of the system (under quasistatic conditions):

$$
K \triangleq \frac{P_{m}}{V_{\mathrm{DAC}}}
$$

Therefore, $K$ is the ratio between the result $\left(P_{m}\right)$ and the electrical command $\left(V_{\mathrm{DAC}}\right)$. One might think about a simple division of the two measurements to get an instantaneous estimation of $K$, but because of noise on both quantities, the result is not exploitable.

A simple and robust way is to use an online estimator of $K$ during the control in real-time. This goal is achieved using a gradient method. An estimator $\hat{K}$ of $K$ is constructed and defined as the solution which minimizes the quadratic error:

$$
\hat{K}=\min _{\bar{K}}\left(\epsilon^{2}(\bar{K})\right) \text { with } \epsilon^{2}(\bar{K})=\left(P_{m}-\bar{K} V_{\mathrm{DAC}}\right)^{2}
$$


A recursive gradient method has been implemented in real-time on the Dspace hardware:

$$
\bar{K}^{(k+1)}=\bar{K}^{(k)}-\gamma \nabla\left(\epsilon^{2}\left(\bar{K}^{(k)}\right)\right),
$$

The convergence of $\bar{K}$ toward $\hat{K}$ is controlled by $\gamma$, a positive real number which should be properly tuned (larger $\gamma$ increases the convergence speed but may also prevent convergence). Note that in the ideal static case, where the servo-valve operates in its linear range, $K$ is proportional to $\frac{P_{m_{0}}}{Q_{l_{0}}}$.

b. Law for the controller tuning Simplifying assumptions have been adopted:

- only the $k_{i}$ parameter is adapted

- $k_{i}$ is varied linearly with respect to the inverse of $\hat{K}$. This law is based on simulated experiments and on the observation of bode diagrams of the open-loop transfer function for different operating points.

Therefore, the tuning of the controller is chosen:

$$
k_{i}=\beta k_{i_{0}} \frac{\hat{K}_{o}}{\hat{K}}
$$

where $k_{i_{0}}$ is the tuning for an arbitrary reference operating point and $\hat{K}_{0}$ the value of the estimator at the same operating point. The proportionality constant $\beta$ is tuned by examining the bode diagrams at the operating point with the most important $P_{m_{0}}$ (to avoid over compensation).

Figure 13 shows the effect of the compensation on a simulated case: the time response of the control has been homogeneised compared to the non-compensated case of figure 12. This is not an optimal correction since the actual pole (which varies with $R_{i}$ ) of the transfer function defined by the process variable time constant is not compensated. One objective should be to compensate for this pole to obtain a perfect homogeneisation. However, it is shown on figure 13 compared to figure 12 that results are already largely improved.

In order to better understand the influence of the adaptative command when the target is a ramp, simulations have been carried out. The complete model is taken into account: servo-valve, volume of the artificial mouth and Bernoulli relation at the inlet of the recorder. The target is a linear ramp which goes from 0 to $3 \mathrm{kPa}$ in $10 \mathrm{~s}$. The controller is either a PI with constant coefficients (left column) or a PI with an integral gain adapted in real time according to Eq. (27) (right column). Results are presented in figure 14. Results show that control performances are more homogenous (as expected) with the adaptative command. Indeed, the asymptotic error (around $0.03 \mathrm{kPa}$ ) is the same but the maximum error is five times smaller with the adaptative command. The simulation has been done with $\gamma=100$ for the online

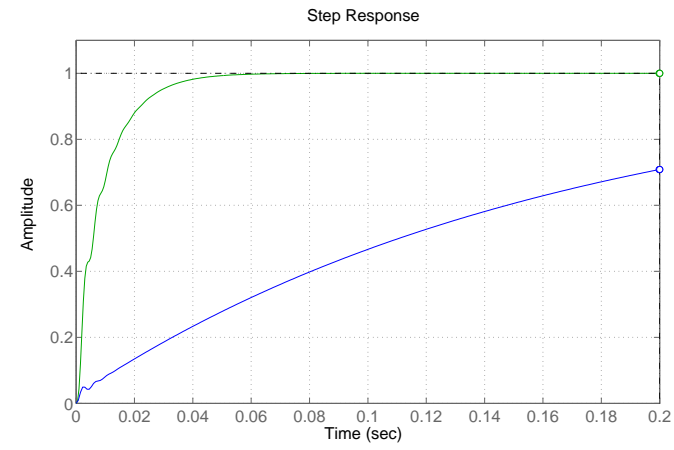

Figure 12. Simulation: step response of the closed loop (Eq. 23) for two operating points of the recorder: 10Pa (blue line) and $3 \mathrm{kPa}$ (green line) and a single PI tuning (tuned at $3 \mathrm{kPa}$ ), showing large disparity.

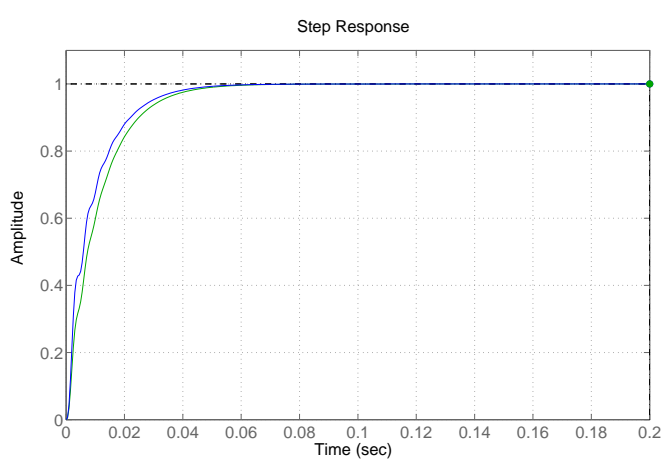

Figure 13. Simulation: step response of the closed loop for two operating points of the recorder: $10 \mathrm{~Pa}$ (blue line) and $3 \mathrm{kPa}$ (green line) when the coefficient $k_{i}$ is adapated according to Eq. (27).

estimator of $K$ (Equ. (26)). A higher $\gamma$ should produce better results, but care has to be taken since a too high $\gamma$ may induce instabilities in the estimation of $\hat{K}$. In that case control performances decrease.

\section{5. "Apparent linearisation" of the friction characteristics}

We have studied in IV A 3 and IV A 4 the tuning of the controller according to expected performances with respect to stability constraints. A major effect which may destabilise the control is the nonlinearity due to internal friction of the servo-valve. This friction yields some stickslip phenomenon which destabilises the system. Several approaches have been considered $\left({ }^{2}\right)$ to try to "linearize" the servo-valve without any identification of a friction model. They correspond to features added to the electrical signal sent to the servo-valve or to an additional control loop based on a second sensor (cascade loop). They have been tested in real time and conclusions are summarized below:

- a dither signal : it is a signal added to the servo- 

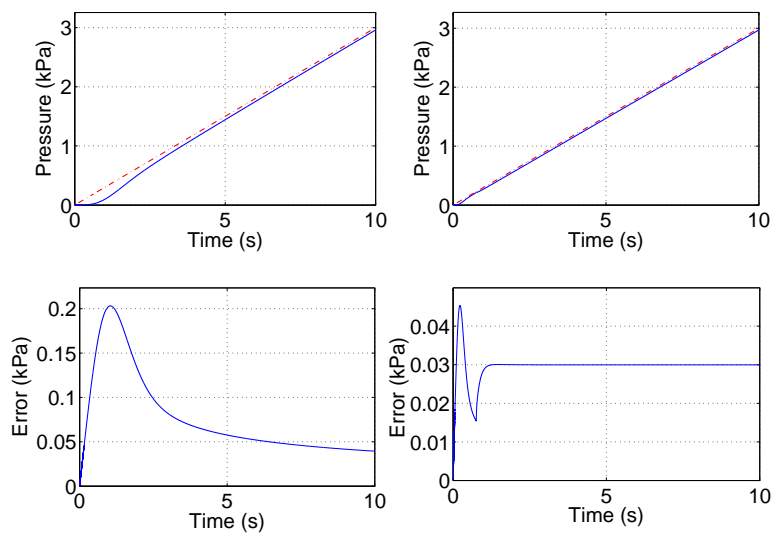

Figure 14. Simulation of the closed loop in the recorder case: influence of the adaptative command on a ramp (left: the controller is a simple PI; right: the integral gain is adapted according to Equ. (27)).

valve input current to "shake" the armature so that it is never stuck. This approach requires many trials to find the optimal dither signal (which depends on the servo-valve mechanical resonance frequency), but once tuned this solution offers good performances in terms of robustness (it works both for constant targets and ramps).

- a feed-forward command : it is also a signal added to the servo-valve input current, but with a different goal: a static value to compensate for inner servo-valve static forces plus a filtered derivative of the command profile to "shake" the armature when the command is changed. It is simple and systematic (automatically generated with respect to the target signal) but it does not work for constant targets (by nature).

- a Coulomb-based offset (a compensation of friction through an offset added to the current sent to the servo-valve): simple and systematic but requires the estimation of the offset (linked to the width of the hysteresis in friction characteristics) and it does not work for constant targets.

Figure 15 shows the effect of a dither signal on the hysteresis reduction of the servo-valve behavior (quasi-static regime). Note on the original characteristic, around $V_{\mathrm{DAC}}=6.8 \mathrm{~V}$, a rapid increase in the volume flow. This is due to stiction effect, with a sudden release of the servo-valve armature. Such phases are used to roughly estimate the resonance characteristics $\left(\omega_{r}, \zeta\right)$ of the armature. When well adjusted (a square signal, with an amplitude equal to the half the width of the original hysteresis, with a frequency close to the armature resonance frequency), the hysteresis decreases drastically. However this result is obtained in quasistatic. Some comparisons are presented in figure 16 when the target is a linear

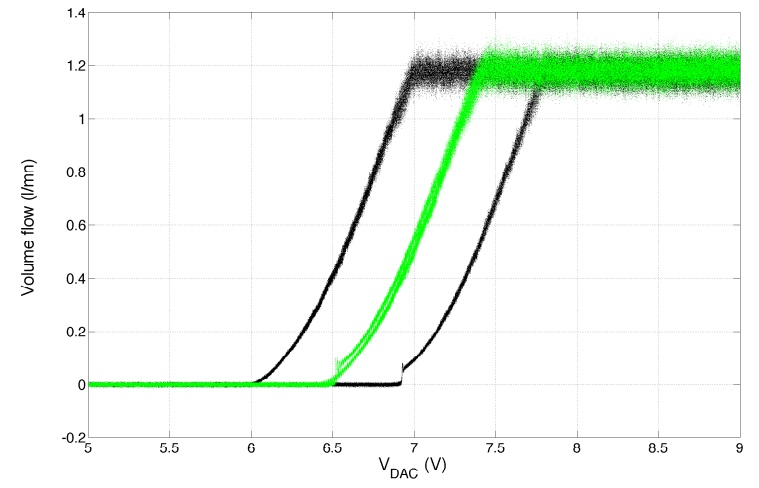

Figure 15. Influence of the dither signal on the reduction of the friction characteristics of the servo-valve (quasi-static regime): without dither (black), with dither (green/gray).

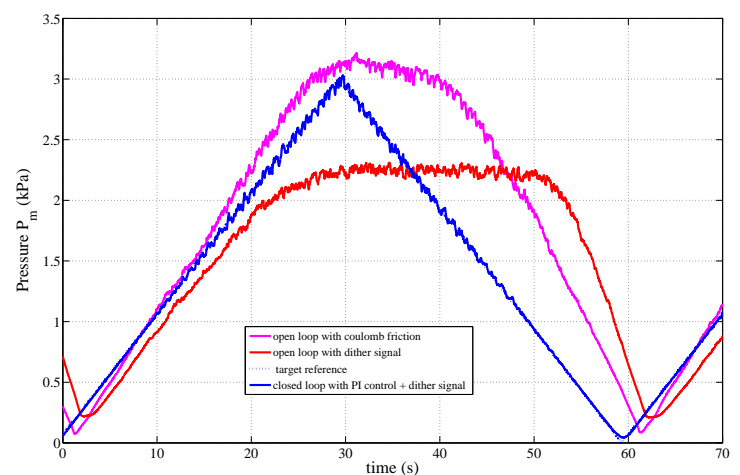

Figure 16. Some approaches tested to compensate the friction of the servo-valve.

ramp. Firstly, dither signal and Coulomb-based compensation are tested in an open-loop scheme. When considering the increasing ramp, results are convincing until the two-third of the ramp, because we stay in the middle range of the valve. Beyond, when approaching the full opening of the servo-valve, efficiency of the dither signal decreases and trajectories are far from the target. However, when associated to the PI controller in a closed loop scheme, the dither signal show very good performances since the trajectory cannot be distinguished on the figure from the target. This solution is adopted.

\section{B. Evaluation of the regulation performance around slowly varying pressure ramps}

Figure 17 shows the performance for a ramp regulation in real conditions, that is when blowing into a recorder. The target mouth pressure goes from 0 to $3 \mathrm{kPa}$ and is masked on the figure by the measured mouth pressure (curves are superimposed). The integral gain is automatically corrected on the basis of the online gain estimation of $K$ (see section IV A 4). The trajectory error remains 


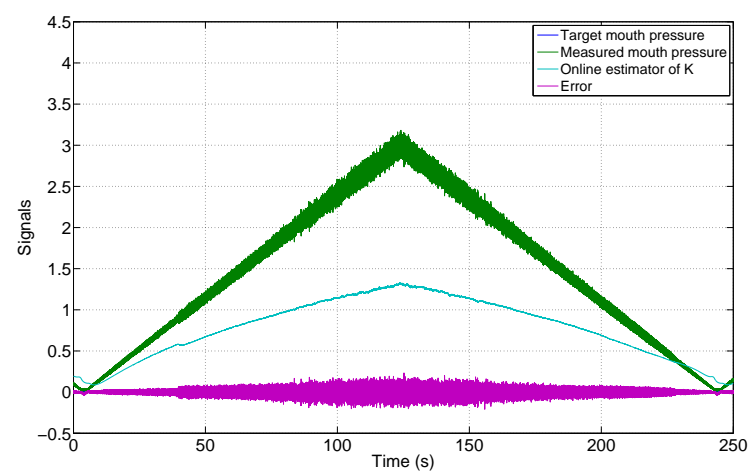

Figure 17. Regulation performances in closed loop, when blowing into a recorder. The controller is a PI with integral gain adapted according to the online estimation of $K$ (see Eq. (27)) and dither signal is added. The target is masked by the measured mouth pressure.

at zero (mean value) from very low to extreme pressures. The increase of the error amplitude is only due to the increase of turbulent noise when the volume flow through the servo-valve is increased. This confirms that the use of an adaptive commant allows to have homogenous performances even on large range of pressures.

\section{CONTRIBUTIONS TO THE STUDY OF RECORDER FUNCTIONNING}

The high-precision regulation is now applied to inspect the functionning of a recorder (a Zen-On "Bressan" alto model). More precisely, it is used to investigate questions hardly tractable without a finely controlled blowing pressure : the transition between two regimes of oscillation when $P_{m}$ is increased, and the focus on low $P_{m}$ which reveals the existence of whistle-tones.

One of the subjects of interest in the study of flute-like instruments is the influence of some small "details" of the geometry on the global behavior of the instrument. For instance, chamfers at the exit of the flue channel are cut with great care by recorder makers. Indeed, adjustements at the scale of tenths of millimeters have a great influence on the dynamical behavior as well as on the sound quality of the recorder. This is well known by makers and players, but somehow difficult to investigate in a scientific context.

In flute-like instruments, the acoustic oscillation is produced by the coupling of the hydrodynamic instability of the air jet with acoustic resonances of the pipe. One of the key points in the oscillation is the ratio of the sound period to the time for the air to fly from the flue exit to the labium. When the jet velocity increases, the travel time of the jet gets smaller compared to the sound period, and eventually the system jumps to the higher pipe resonance. Coming back to lower jet velocities, the system jumps back to a lower pipe resonance, but for a velocity lower than for the increasing path: the system

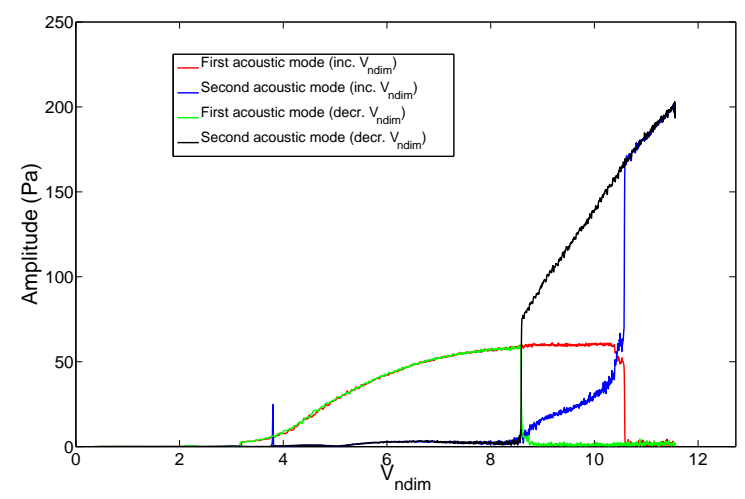

Figure 18. Transition diagram for a low G fingering on a Zen-On recorder, using the standard channel and chamfer geometry.

shows an hysteresis. Both the velocity for regime transition and the width of the hysteresis are important for the player: increasing the transition velocity allows the player to play louder on a given regime, while increasing the width of the hysteresis offers a greater stability of the instrument.

In experiments, the precise determination of regime transitions is usually one of the difficulties. Indeed, fluctuations of the blowing pressure $P_{m}$ cannot be avoided without a closed loop control, and consequences on the experimental results can hardly be quantified when the instrument is close to a bifurcation. This transition has been discussed by Gibiat $\left(^{4}\right)$, suggesting the possibility of a blowing range in which two oscillating regimes can coexist in a bi-periodic oscillation, or even a chaotic oscillation. The transition diagram is presented in figure 18 in the case of the Zen-On "Bressan" alto recorder, fingering low $\mathrm{G}(\mathrm{G} 4,392 \mathrm{~Hz})$. The pressure inside the recorder is analyzed in terms of its frequency components. Figure 18 shows the evolution of the two first frequency components corresponding to the two first pipe modes, for a blowing pressure following a slow (120s) increase/decrease ramp. The amplitude of the frequency components are plotted as function of the dimensionless jet velocity $V_{n d i m}=U_{j} / f_{1} W$, where $U_{j}$ is the jet velocity estimated from the measured pressure using Equ. (14), $f_{1}$ is the first pipe mode frequency, and $W$ is the distance from flue exit to labium. A short-term Fourier transform is used to separate the frequency components, with a resolution of one period $1 / f_{1}$ of the first mode.

Figure 18 shows that the transition from first to second oscillation regime appears for an increasing dimensionless jet velocity of 10.6 , easily detected by the sudden drop of the amplitude of the first frequency component accompanied simultaneously by a sudden increase of the amplitude of the second component. The transition down from second to first regime appears at a decreasing dimensionless jet velocity of 8.6 . The high precision regulation 


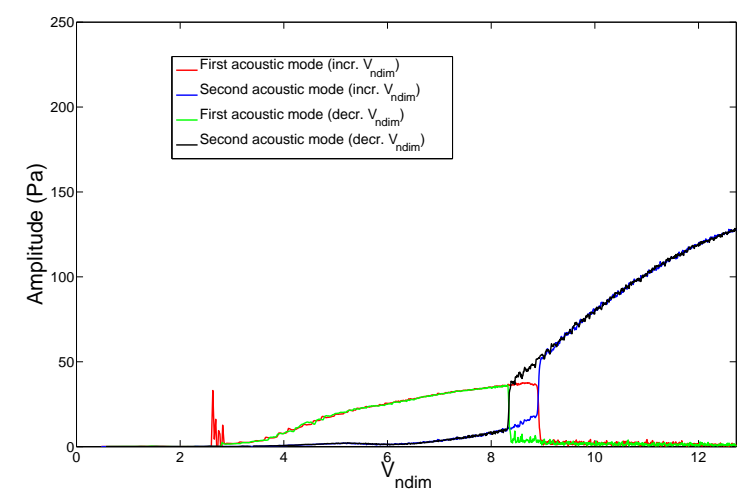

Figure 19. Transition diagram for a low G fingering on a Zen-On recorder, using a straight channel without chamfers.

allows to show that no bi-periodic nor chaotic regimes appear in the transitions.

The geometry of the channel and chamfers is now changed, using a straight channel without chamfers at the end (see figure 19). This upgoing (resp. downgoing) transition appears now for a dimensionless jet velocity of 8.9 (resp. 8.4) instead of 10.6 (resp. 8.6) with the orginal geometry. Hence we can evaluate the influence of the maker's work and the important consequences on the possibilities offered to the player: with the original chamfers, the instrument can sound louder (on both regimes) and the transition between the two regimes is safer (larger hysteresis width).

Figures 18 and 19 also show interesting phenomena for low values of the dimensionless jet velocity $\left(V_{\text {ndim }} \approx\right.$ $3 \ldots 4)$. The oscillation appears on for a very short range of blowing velocity. This corresponds to the higher hydrodynamic modes as discussed by Coltman $\left({ }^{5}\right)$ and characterised by very low values of the inverse Strouhal number $U_{j} / f W$. Theses regimes are known as "whistletones" also called "aeolian sounds" and produce a weak sound due to the low jet velocity. These "whistle-tones" may appear on any of the acoustic resonances of the pipe and are characterised by the combination of a higher hydrodynamic mode and a specific acoustic mode : on the first acoustic mode on figure 18 and on the second acoustic mode on figure 19. They are well known in the organ where they are easily heard when the ventilator is turned off letting the reservoir pressure fall slowly $\left({ }^{6},{ }^{7}\right)$. Because of the relatively short distance $W$ from flue exit to labium in the recorders, these specific regimes were never observed in such instruments. Their playing pressure range is so small that only the high-precision regulation allows to identify such regimes in the recorder.

Finally, figure 20 presents the spectrogram of the acoustic pressure in the recorder for a blowing pressure following a slow (120s) increase/decrease ramp. The main difference with the previous case lies in the fact that the different pipe modes are not close to a harmonic as was the case for the low $\mathrm{G}$ fingering. Figure 20 shows

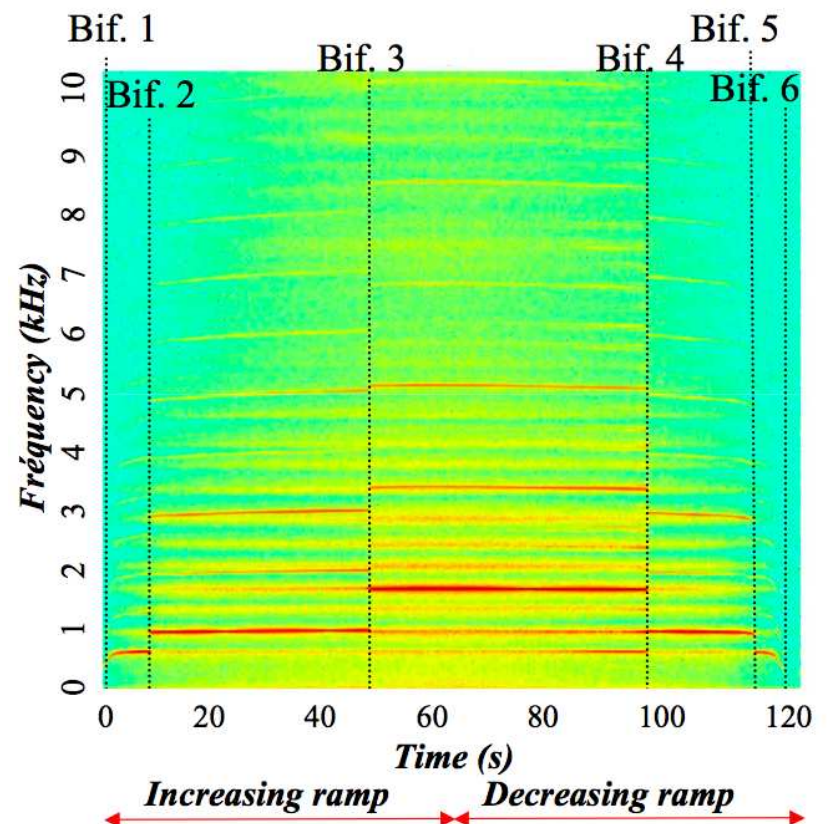

Figure 20. Spectrogram of the inner acoustic pressure for a E flat fingering on a Zen-On Bressan alto recorder, using standard channel and chamfer geometry, for a slow increasing / decreasing blowing pressure ramp.

that even in this case, there is no evidence of bi-periodic regimes, in which the non-linearity of the acoustic sources in the recorder would produce cross product terms with sum and difference frequency components.

\section{CONCLUSION}

The closed-loop strategy presented in this paper is a direct improvement of what is usually done "by hand" when working with an artificial mouth: the operator has the hand on the pressure reducer and an eye on the manometer, in order to react as fast as possible and reduce the gap between the target and the observed pressure. For constant mouth pressure targets, we could achieve the regulation of the mean pressure with an error around $1 \mathrm{~Pa}$. When considering slow ramps, the use of an adaptive command allows to have homogenous performances even on a large range of blowing pressure (as shown in section IV B).

The high-precision regulation proved to be a valuable tool for the acoustician in order to inspect the behavior of the instrument around precise transitions between regimes or on large range of blowing pressures. According to the preliminary investigations presented in this paper, it looks like a promising way of studying the influence of the work of the instrument maker.

However it should be noticed that the solution developped in this paper cannot be automatically re-used for all the instruments. Indeed, the question of the controller architecture should be investigated for each different fam- 
ily of instruments. For instance our short-term perspectives include the study of pressure regulation for single reed instruments and preliminary investigations suggest typical difficulties due to non monotonous relation between the mouth pressure and the volume flow blown into the instrument. This will be detailed in a forthcoming paper.

\section{Acknowledgements:}

This study was lead with the support of the French National Research Agency ANR within the CONSONNES project. The experimental setup has been manufactured at LMA by A. Busso and S. Devic.

${ }^{1}$ C.S. McGinnis and C. Gallagher, The mode of vibration of a clarinet reed, J. Acoust. Soc. Amer., Vol. 12, pp529-531, 1941.
${ }^{2}$ H. Olsson and K. J. Aström and C. Canudas de Wit and M. Gäfvert and P. Lischinsky, Friction Models and Friction Compensation. European Journal of Control, Vol. 4, pp.176195, 1998.

${ }^{3}$ A. Gelb and W. Vander Velde, Multiple input Describing function and Nonlinear System Design, MC Graw Hill, 1968. ${ }^{4} \mathrm{~V}$. Gibiat, Phase space reconstruction of musical signals, Journ. of Sound and Vibration, Vol. 123(3), p529-536,1988.

${ }^{5}$ Coltman, Sounding Mechanism of the flute and organ pipe, J. Acoust. Soc. Amer., Vol. 44(4), 1968.

${ }^{6}$ A. H. Benade and J. W. French, Analysis of the flute head joint, J. Acoust. Soc. Amer., Vol. 37, p679-691, 1965

${ }^{7} \mathrm{H}$. Bouasse, Tuyaux et résonateurs, librarie Delagrave, Paris, 1929

${ }^{8}$ D. Ferrand and C. Vergez, Blowing machine for wind musical instrument : toward a real-time control of the blowing pressure, 16th IEEE Mediterranean Conference on Control and Automation (MED), Ajaccio, France, 2008. 